\title{
Flexible Cystoscopy in the Setting of Macroscopic Hematuria: Do the Findings Justify Its Use?
}

\author{
Reuben Ben-David ${ }^{a, b}$ Samuel Morgan ${ }^{b}$ Ziv Savin ${ }^{a, b}$ Snir Dekaloa,b \\ Mario Sofer ${ }^{a, b}$ Avi Beri ${ }^{a, b}$ Ofer Yossepowitch ${ }^{a, b}$ Roy Mano ${ }^{a, b}$ \\ aDepartment of Urology, Tel Aviv Sourasky Medical Center, Tel-Aviv, Israel; bSackler Faculty of Medicine, Tel Aviv \\ University, Tel-Aviv, Israel
}

\section{Keywords}

Hematuria - Cystoscopy - Urological diagnostic techniques . Urinary bladder neoplasms

\begin{abstract}
Background: Patients hospitalized due to gross hematuria frequently complete evaluation in the outpatient setting. The use of office flexible cystoscopy during hospitalization may lead to prompt diagnosis and treatment but can be limited due to low visualization and artifacts that can hamper diagnostic ability. Objective: The objective of this study was to assess flexible cystoscopy findings and yield performed in patients hospitalized due to gross hematuria. Methods: Medical records of patients who underwent flexible cystoscopy while hospitalized during September 2018-December 2019 were reviewed. Cystoscopic findings were categorized into (1) suspicious mass in the bladder or prostate, (2) nonsuspicious changes in the bladder, and (3) nondiagnostic exam. Descriptive statistics were used to report the clinical characteristics of the study cohort and the findings of cystoscopy. Univariate logistic regression analyses were used to identify predictors of malignant findings. Results: The study cohort consisted of 69 patients (median age of 76 years). Initial cystoscopy findings were suspicious for malignancy in $26 / 69$ patients (38\%), nonsuspicious for malignancy in $34 / 69$
\end{abstract}

karger@karger.com www.karger.com/uin

Karger $\stackrel{\text { ' }}{5}$
C 2021 The Author(s).

Published by S. Karger AG, Basel

This is an Open Access article licensed under the Creative Commons Attribution-NonCommercial-4.0 International License (CC BY-NC) (http://www.karger.com/Services/OpenAccessLicense), applicable to the online version of the article only. Usage and distribution for commercial purposes requires written permission. patients (49\%), and nondiagnostic in 9/69 patients (13\%). The median follow-up time was 9 months (range 4-14 months). Twenty patients (29\%) were diagnosed with malignancy (sensitivity of $75 \%$ and specificity of $78 \%$ ). The procedure led to either diagnosis or treatment of 39 patients (57\%). However, in 30 patients (43\%), the initial cystoscopy did not aid in the diagnosis, led to misdiagnoses, or required a follow-up cystoscopy. On univariate analyses, none of the precystoscopy variables were predictive of bladder malignancy. Conclusion: Flexible cystoscopy in the setting of acute hematuria requiring hospitalization did not lead to diagnosis or treatment in over $40 \%$ of cases. In this setting, consideration should be given to performing an upfront cystoscopy under anesthesia.

(c) 2021 The Author(s).

Published by S. Karger AG, Basel

\section{Introduction}

Gross hematuria is a commonly encountered symptom that may indicate an underlying urologic malignancy [1]. The evaluation of gross hematuria requires the use of cystoscopy, supplemented by urine cytology or additional biomarkers and upper urinary tract imaging [2-4]. Cystoscopy is routinely used for the detection of malig- 
nancies within the bladder $[5,6]$, with sensitivities ranging from 70 to $100 \%$ and a specificity of approximately $70 \%$ [6-9]. Patients presenting with gross hematuria are found to have a demonstrable cause in $50 \%$ of the cases [2], and in $19-34 \%$ of cases, an underlying malignancy is found [10-12].

Patients hospitalized due to acute gross hematuria are commonly treated conservatively with continuous bladder irrigation [13]. In severe (e.g., hemodynamically unstable) and intractable hematuria, patients are taken inevitably to the operating room for transurethral cystoscopy, clot evacuation, a biopsy of suspicious masses, and fulguration of bleeding sources. Because hematuria workup is frequently carried out in the outpatient setting [11], admitted patients in whom the hematuria episode had resolved are often discharged from the hospital without a definitive diagnosis or a treatment plan until further evaluation in the outpatient clinic. This, in turn, may increase the risks associated with treatment delay [14].

While the use of office flexible cystoscopy may lead to prompt diagnosis, in the setting of acute severe hematuria, it is often limited due to commonly encountered changes related to continuous bladder irrigation or the presence of an indwelling catheter (e.g., bullous changes, edematous changes, and artifacts). These findings might lead to a false-positive outcome resulting in unwarranted treatment or, even more concerning, mistaken for benign urothelium in the presence of an underlying malignancy. Moreover, most studies evaluating the use of flexible cystoscopy for hematuria workup include patients who are examined in the non-acute outpatient setting rather than patients who are admitted to the hospital due to acute hematuria $[11,15]$. Therefore, there are limited data regarding the optimal timing of cystoscopic evaluation in patients admitted to the hospital due to severe ongoing hematuria and whether the findings and yield of flexible cystoscopy in this acute setting justify its use.

In the current study, we sought to evaluate the findings and the yield of flexible cystoscopy performed during admission in patients with acute hematuria requiring hospitalization. In addition, we evaluated whether precystoscopy characteristics may predict the presence of an urothelial malignancy of the bladder.

\section{Materials and Methods}

After institutional review board approval, the medical records of 102 hospitalized patients who underwent cystoscopy in the acute setting during September 2018-December 2019 were reviewed, retrospectively. Cystoscopy was performed in 33 patients

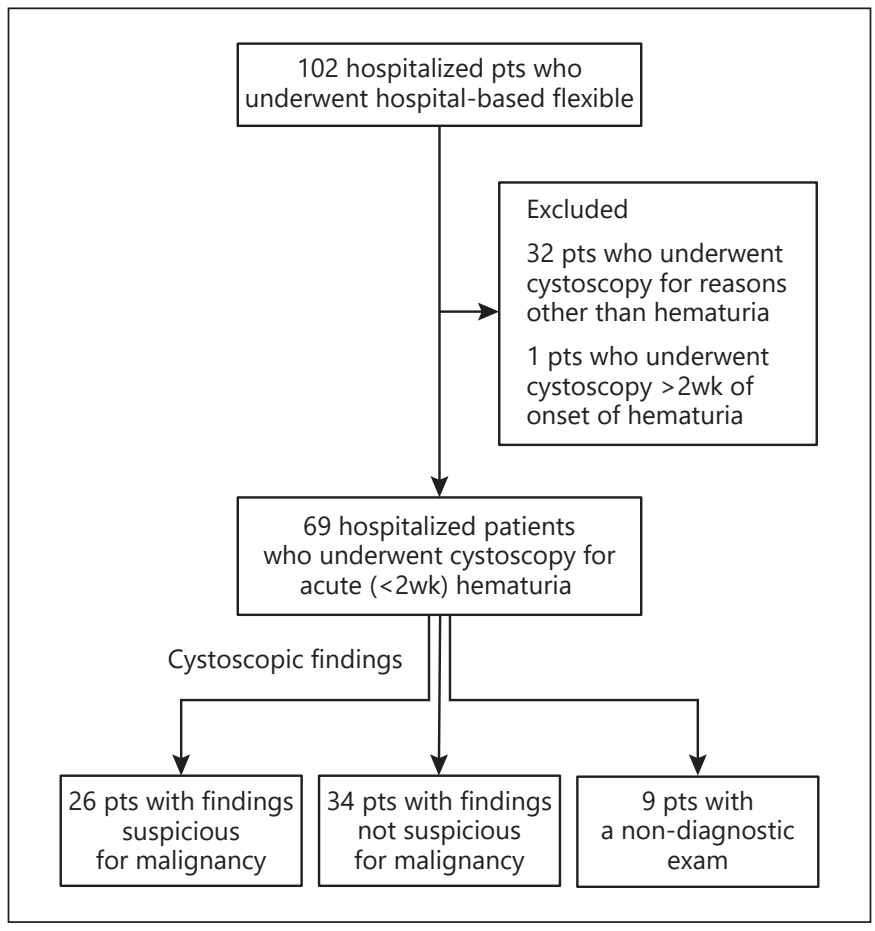

Fig. 1. Flowchart describing the study cohort.

for reasons other than hematuria, including evaluation of an incidentally detected bladder lesion on an imaging study $(n=16)$, evaluation of urinary retention $(n=9)$, and recurrent UTI or dysuria $(n=7)$; these patients were excluded (shown in Fig. 1). One patient who underwent cystoscopy 3 weeks after onset of hematuria was also excluded. A total of 69 patients who underwent cystoscopy for new-onset hematuria requiring hospitalization $<2$ weeks of symptom initiation were included in the final analysis.

Baseline clinical characteristics of the study cohort including age, gender, past surgical and medical history, and medications used (antiplatelets, anticoagulation, $\alpha$-blockers, and 5- $\alpha$ reductase inhibitors) were collected from the patients' medical records. Upper tract imaging was routinely performed as part of the hematuria evaluation during the admission or recommended at discharge. Patients were advised to undergo urine cytology after their discharge.

Cystoscopy was performed with the patient in a lithotomy position using a flexible video cystoscope with saline irrigation. All the procedures were done by a senior urologist or a resident, supervised by a senior urologist. In the case of blood clots obscuring visualization, an attempt was made to evacuate the clots with a Tiemann $18 \mathrm{~F}$ catheter followed by repeat flexible cystoscopy. Cystoscopic findings were categorized as (1) suspicious mass in the bladder or prostate, (2) bullous/hyperemic changes in the bladder, (3) bullous/hyperemic changes in the prostate, (4) normal cystoscopy, and (5) nondiagnostic exam. Based on these findings, the outcome was further categorized as (1) suspicious for malignancy, (2) findings not suspicious for malignancy, and (3) nondiagnostic.

After the initial cystoscopy, patients were followed routinely, and findings of additional cystoscopies were recorded. Pathology 
Table 1. Baseline characteristics of patients included in the study cohort categorized by the results of initial cystoscopy

\begin{tabular}{|c|c|c|c|}
\hline Characteristic & $\begin{array}{l}\text { Suspicious mass } \\
(n=26)\end{array}$ & $\begin{array}{l}\text { Findings not suspicious } \\
\text { for malignancy }(n=34)\end{array}$ & $\begin{array}{l}\text { Nondiagnostic } \\
(n=9)\end{array}$ \\
\hline Age (median, IQR), years & $77(65-86)$ & $76(67-85)$ & $70(65-85)$ \\
\hline \multicolumn{4}{|l|}{ Gender, $n(\%)$} \\
\hline Male $(n=54)$ & $21(81)$ & $25(73)$ & $8(89)$ \\
\hline Female $(n=15)$ & $5(19)$ & $9(26)$ & $1(11)$ \\
\hline Blood thinners (yes, $n=46), n(\%)$ & $16(61)$ & $23(68)$ & $7(78)$ \\
\hline $\mathrm{AP}$ & 13 & 18 & 5 \\
\hline $\mathrm{AC}$ & 2 & 4 & 2 \\
\hline $\mathrm{AP}+\mathrm{AC}$ & 1 & 1 & 0 \\
\hline \multicolumn{4}{|l|}{ Medical history of } \\
\hline $\mathrm{BPH}(n=13)$ & 2 & 9 & 2 \\
\hline Post-TURP $(n=11)$ & 5 & 6 & 0 \\
\hline $\operatorname{TCC}(n=6)$ & 2 & 3 & 1 \\
\hline $\operatorname{CKD}(n=6)$ & 1 & 5 & 0 \\
\hline Pelvic irradiation $(n=7)$ & 4 & 2 & 1 \\
\hline Prostate cancer $(n=5)$ & 3 & 1 & 1 \\
\hline Recurrent UTI $(n=5)$ & 1 & 4 & 0 \\
\hline a-Blocker (yes), $n(\%)$ & $3(11)$ & $14(41)$ & $6(67)$ \\
\hline 5-a Reductase inhibitors (yes), $n$ (\%) & 0 & $4(12)$ & $2(22)$ \\
\hline
\end{tabular}

$\mathrm{CKD}$, chorionic kidney disease; AP, antiplatelet medication; AC, anticoagulation medication; UTI, urinary tract infection.

Table 2. Summary of cystoscopy findings as classified by the urologists who performed the procedure ( $>1$ finding can be described per patient)

\begin{tabular}{lccc}
\hline Cystoscopic findings & $\begin{array}{l}\text { Suspicious } \\
\text { mass } \\
(n=26)\end{array}$ & $\begin{array}{l}\text { Findings not } \\
\text { suspicious for } \\
\text { malignancy } \\
(n=34)\end{array}$ & $\begin{array}{l}\text { Nondiagnostic } \\
(n=9)\end{array}$ \\
\hline Normal findings & 0 & 9 & 0 \\
Suspicious mass & 26 & 0 & 0 \\
Hyperemic prostate & 3 & 9 & 4 \\
Edematous/bullous changes within the bladder & 5 & 21 & 3 \\
Severe hematuria, bladder changes limiting diagnostic ability & 0 & 0 & 9 \\
\hline
\end{tabular}

specimens, when available, were evaluated by a dedicated genitourinary pathologist and staged according to the TNM staging system.

Study findings were reported using descriptive statistics. Categorical variables were reported as frequency and percentage, and continuous variables were reported as the median and interquartile range. The Fisher exact test was used to compare categorical values, and the Mann-Whitney test was used to compare medians. Univariable logistic regression analyses were used to evaluate the association between baseline characteristics, prior to cystoscopy, and the presence of a bladder tumor at the completion of the hematuria evaluation. Neutrophil lymphocyte ratio (NLR) and platelet lymphocyte ratio (PLR) were previously associated with the presence and aggressiveness of bladder tumors and were therefore

Findings of Flexible Cystoscopy in the Setting of Acute Hematuria evaluated as additional predictors for identifying a bladder tumor at the completion of evaluation [16]. A $p$ value of $<0.05$ was considered statistically significant. SPSS was used for all statistical analyses (IBM SPSS statistics for windows, version 25; IBM Corp, Armonk, New York, NY, USA).

\section{Results}

The study cohort consisted of 69 patients (54 males and 15 females) at a median age of 76 years (IQR: $67-85$ ). Baseline clinical characteristics of the study cohort are re- 


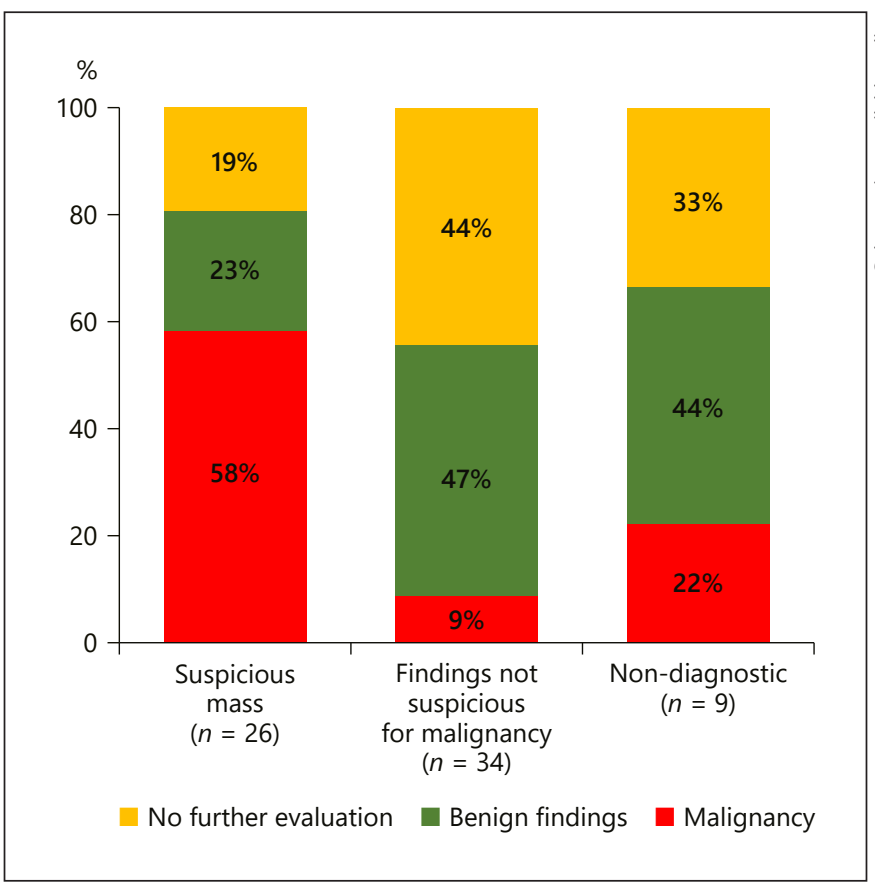

Fig. 2. Findings at the end of follow-up stratified by results of the initial cystoscopy.

ported in Table 1. The median time between the onset of hematuria and cystoscopy was 3 days (IQR: 1-7). Findings on initial cystoscopy are reported in Table 2 and shown in Figure 2; 18 patients (26\%) had $>1$ finding during cystoscopy. A total of 26 patients (38\%) had findings suspicious for malignancy. Thirty-four patients had nonspecific changes without clear evidence of malignancy (49\%), and 9 patients (13\%) had a nondiagnostic exam (shown in Fig. 1). All patients had a minimum follow-up of 4 months after the initial cystoscopy, and the median follow-up time was 9 months (range 4-14 months). Upper tract imaging was available during the admission for 41 patients (4 had imaging studies performed within 3 months prior to the admission and 37 performed imaging studies during their admission). The remaining $28 \mathrm{pa}-$ tients were recommended to undergo imaging in the outpatient setting. During the follow-up period, no upper tract or renal tumors were detected.

All 26 patients with suspicious mass/findings were offered transurethral resection of the tumor under general anesthesia. A total of 21/26 underwent TURBT, and malignancy was found in $15(22 \%)$ patients (8 had highgrade bladder cancer, 6 low-grade bladder cancer, and 1 was diagnosed with ISUP-5 prostate cancer). In 6 patients (9\%), benign bladder changes without malignancy were found. Among the 5 patients who did not undergo a TURBT, 3 were medically unfit, 1 refused surgery, and 1 was lost to follow-up.

Of the 34 patients with findings that were not initially suspicious for malignancy, 16 patients were referred to follow-up cystoscopy and 7 underwent surgery. One (3\%) was diagnosed with HG urothelial carcinoma of the bladder, whereas the other 6 (18\%) had transurethral prostatectomy for BPH; 9 patients had a follow-up cystoscopy, and 2 high-grade bladder malignancies were found (6\%). The remaining 18 patients were not referred for additional follow-up, and no malignancies were found during the follow-up period.

Nondiagnostic cystoscopies were reported in 9 patients; of those, 4 were referred to cystoscopy under anesthesia, 2 were found to have HG TCC and 2 underwent TURP with BPH on pathology; 2 had a repeated cystoscopy with normal findings; 2 died in proximity to the hematuria episode; and 1 was lost to follow-up.

Altogether, of the 69 cystoscopies performed to evaluate hematuria in the acute setting, 20 malignancies (29\%) were diagnosed, including 19 bladder tumors and 1 highrisk prostate cancer. Only 15 of these cases (75\%) were identified based on the initial cystoscopy, whereas the other 5 were diagnosed during further follow-up. Thus, within the setting of acute hematuria, the sensitivity and specificity of cystoscopy for identifying malignancy were $75 \%$ and $78 \%$, respectively. Furthermore, the initial cystoscopy examination required $15(22 \%)$ follow-up cystoscopies.

For the evaluation of precystoscopy predictors of a bladder tumor, 2 patients with known hematologic malignancies were excluded from the analyses. Of the 67 remaining patients, 19 patients (28\%) were diagnosed with a bladder tumor. NLR was higher among patients who were diagnosed with a bladder tumor (4.57 vs. 3.71); however, this did not reach statistical significance $(p=0.487)$. On univariable analyses, none of the preoperative characteristics were significantly associated with the presence of a malignancy (Table 3).

\section{Discussion}

In the current study, the diagnostic yield of flexible cystoscopy performed in 69 patients hospitalized due to acute severe hematuria was evaluated. Within this setting, 26 patients (38\%) had a lesion suspicious for malignancy, 15 of whom (58\%) were found to have a malignancy on subsequent evaluation ( $75 \%$ sensitivity and $78 \%$ specific- 
Table 3. Univariable logistic regression analyses of precystoscopy predictors of bladder malignancy during hematuria evaluation

\begin{tabular}{lrrrr}
\hline Variable & Odds ratio & $2.50 \%$ & $97.50 \%$ & $p$ value \\
\hline Age (per 1 year) & 1.015 & 0.97 & 1.064 & 0.519 \\
Male sex & 1.013 & 0.248 & 3.568 & 0.984 \\
Blood thinner use & 1.188 & 0.392 & 3.897 & 0.766 \\
History of prostatic hypertrophy & 0.447 & 0.064 & 1.935 & 0.331 \\
History of urothelial cancer & 0.689 & 0.096 & 3.209 & 0.662 \\
History of pelvic radiation & 1.294 & 0.168 & 7.289 & 0.777 \\
History of prostate cancer & 2.812 & 0.479 & 16.6 & 0.233 \\
Neutrophil count & 1.063 & 0.9 & 1.254 & 0.459 \\
Lymphocyte count & 0.785 & 0.37 & 1.279 & 0.452 \\
Platelet count & 1 & 0.994 & 1.006 & 0.931 \\
NLR & 1 & 0.883 & 1.108 & 0.998 \\
PLR & 0.999 & 0.994 & 1.005 & 0.806 \\
\hline
\end{tabular}

NLR, neutrophil lymphocyte ratio; PLR, platelet lymphocyte ratio. ity). Among the 43 patients with nonspecific changes within the bladder or a nondiagnostic exam, 14 patients eventually required an additional cystoscopy under anesthesia, while 29 patients avoided a procedure under anesthesia during follow-up. Malignancy rates were higher in the nondiagnostic group (22\%) compared to the nonsuspicious group (9\%), a difference that may be attributed to the better visualization in the nonsuspicious group. However, even within the nonsuspicious group, malignancy rates were nonnegligible.

Edwards et al. [15]reported on a large cohort of 687 patients who underwent cystoscopies in the setting of an outpatient hematuria clinic. The overall prevalence of malignant disease for patients presenting with macroscopic hematuria was $19 \%$, whereas the probability of missing a malignancy was $2-4 \%$. The results of the current study show a slightly higher rate of diagnosing malignancies (26\%), while the rate of missing a malignancy on cystoscopy was comparable (3\%). Our higher malignancy rates may be associated with the different study population, as our patients required hospitalization due to their hematuria. In addition, we observed a relatively high proportion of patients who received antithrombotic medications $(67 \%)$ resulting in earlier hematuria episode and tumor detection $[17,18]$.

A possible benefit of performing a cystoscopy in the acute setting when a patient is admitted for hematuria is early detection of the cause of hematuria. This may, in turn, limit the diagnosis delay which has been reported previously by Fahmy et al. [14] and Gore et al. [19], who found an increased mortality rate when treatment was delayed for $>3$ months in patients with muscle invasive disease. In institutes with limited access to the operating room, implementing different cystoscopy techniques may improve the yield of office cystoscopy, overcoming the limitations observed in our study. Previous reports suggest the use of air cystoscopy can improve diagnostic ability [20]. In a study by Ciudin et al. [21], the use of air cystoscopy was compared to that of water cystoscopy in patients with and without active hematuria. In a subgroup of 57 patients who underwent cystoscopy during active hematuria, the cause of hematuria was identified twice as much using air cystoscopy than water cystoscopy ( 86 vs. $38 \%$, respectively, $p=0.001$ ), and air cystoscopy had an $88 \%$ sensitivity and $97 \%$ specificity which was superior to those of water cystoscopy (47\% sensitivity and $100 \%$ specificity) [21]. In the current study, the use of water cystoscopy was associated with a $75 \%$ sensitivity and $78 \%$ specificity, both of which were lower than those reported for air cystoscopy. Thus, future studies should evaluate whether in cases with undetermined findings on water cystoscopy, complementing the exam with air cystoscopy can improve the diagnostic characteristics of the exam.

In cases of ongoing hematuria, cystoscopy under anesthesia and resection/fulguration of lesions may be needed [2]. The results of this study suggest that approximately half of the cohort would have benefited from an evaluation under anesthesia, rather than an initial flexible cystoscopy. This approach could also decrease the complications associated with ongoing hematuria and blood loss as well as the long period of time that a patient is worn off from antithrombotic medications and may avoid the need for multiple procedures. The drawback of performing cystoscopy under anesthesia for all patients is the increase in unnecessary pro- 
cedures with anesthesia due to lower specificity. The high rate of additional procedures required should therefore be discussed with the patient prior to performing flexible cystoscopy within the setting of acute hematuria requiring hospitalization.

Finally, no precystoscopy characteristics which could predict the presence of a malignancy at the completion of hematuria evaluation were found. Previous studies suggest that patients with hematuria have an increased rate of genitourinary malignancies if they are older, suffer from macrohematuria rather than microhematuria, are of male sex, and were exposed to tobacco or occupational toxins [22]. Multiple studies have shown an association between bladder cancer and NLR. Pretreatment NLR is a useful biomarker for disease aggressiveness and treatment response in patients with bladder cancer [16]. In the current study, we aimed to evaluate whether pretreatment NLR and PLR would be associated with the finding of a malignancy during evaluation. While NLR was higher among patients found to have a bladder tumor, this finding was not statistically significant. Future studies on larger cohorts should evaluate whether NLR can predict malignancy in patients with hematuria.

The retrospective nature of this study carries inherent limitations. First, the relatively small cohort size presents a limitation. Nonetheless, our findings raise important questions about the yield of flexible cystoscopy in the acute setting which may affect its use in the management of patients admitted to the hospital due to hematuria. Future studies with larger cohorts are required to validate our findings prior to implementing them into clinical practice. Second, due to the relative short follow-up period of 9 months no long-term findings have been evaluated; nevertheless, the main purpose of the study was to evaluate the outcomes of the initial cystoscopy rather than the long-term outcomes of hematuria that have been well described in several other studies $[1,11]$. Third, the cystoscopy findings were described by different urologists; thus, there may have been inconsistencies in diagnosis or treatment plan based on the experience and preference of the different urologists as encountered in other countries [23]. Some of the nonspecific changes seen in the bladder as well as the nondiagnostic nature of some of the procedures might be a direct result of the short time to cystoscopy from the onset of the acute hematuria episode (median of 3 days). Although all the cystoscopies were performed when the urine was clear, delaying the procedure may have aided by seeing fewer artifacts and clots; however, we were unable to determine the optimal timing for performing cystoscopy based on the results of the current study. Finally, 8 patients did not undergo a surgical procedure or a repeated cystoscopy despite an initial recommendation to repeat the procedure ( 5 medically unfit, 1 refused, and 2 lost to follow-up), in 5 of whom a suspicious mass was found; as a result, their final pathology might have altered our results.

In conclusion, hospital-based cystoscopy carries both inherent advantages and disadvantages, the advantage being mainly the ability to achieve a prompt diagnosis and an acceptable rate of diagnostic yield (55\%), while the drawbacks include the need for additional cystoscopy in a substantial group of patients. In centers where the operating room is widely available, consideration should be given to performing the initial cystoscopy under anesthesia and intervening accordingly; otherwise, a short delay ( 2 weeks) and outpatient setting cystoscopy may be performed instead. Future studies should evaluate whether modified cystoscopy techniques, such as the use of air cystoscopy, may improve the diagnostic yield of the procedure in this setting.

\section{Statement of Ethics}

The study protocol was approved by the Ethics (Helsinki) Committee of the Tel-Aviv Medical Center, \#0068-20-TLV (June 2020).

\section{Conflict of Interest Statement}

The authors declare no conflicts of interest.

\section{Funding Sources}

The authors received no financial support for this research.

\section{Author Contributions}

R.B-D. conceived the idea and study design, analyzed the data, and led manuscript writing; Z.S., S.M., and S.D. assisted with study design and data collection; M.S., A.B., O.Y., and R.M. audited data collection integrity and provided consult and guidance in manuscript writing. All authors have reviewed and approved this version of the manuscript. 


\section{References}

1 Nørgaard M, Veres K, Ording AG, Djurhuus JC, Jensen JB, Sørensen HT. Evaluation of hospital-based hematuria diagnosis and subsequent cancer risk among adults in Denmark. JAMA Netw open. 2018;1:e184909.

2 Wein AJ, Kavoussi LR, Partin AW, Peters CA. Campbell-Walsh urology. 11th ed. Elsevier; 2015.

3 Davis R, Jones JS, Barocas DA, Castle EP, Lang EK, Leveillee RJ, et al. Diagnosis, evaluation and follow-up of asymptomatic microhematuria $(\mathrm{AMH})$ in adults: AUA guideline. J Urol. 2012;188:2473-81.

4 Sathianathen NJ, Butaney M, Weight CJ, Kumar R, Konety BR. Urinary biomarkers in the evaluation of primary hematuria: a systematic review and meta-analysis. Bladder Cancer. 2018;29:353-363.

5 Chang SS, Boorjian SA, Chou R, Clark PE, Daneshmand S, Konety BR, et al. Diagnosis and treatment of non-muscle invasive dladder cancer: AUA/SUO guideline. J Urol. 2016.

6 Alfred Witjes J, Lebret T, Compérat EM, Cowan NC, De Santis M, Bruins HM, et al. Updated 2016 EAU guidelines on muscle-invasive and metastatic bladder cancer. Eur Urol. 2017;71:462-75.

7 Mitropoulos D, Kiroudi-Voulgari A, Nikolopoulos P, Manousakas T, Zervas A. Accuracy of cystoscopy in predicting histologic features of bladder lesions. J Endourol. 2005;19:861-4.

8 Zhu CZ, Ting HN, Ng KH, Ong TA. A review on the accuracy of bladder cancer detection methods. J Cancer. 2019;10:4038-44.
9 Ye Z, Hu J, Song X, Li F, Zhao X, Chen S, et al. A comparison of NBI and WLI cystoscopy in detecting non-muscle-invasive bladder cancer: a prospective, randomized and multicenter study. Sci Rep. 2015;5:10905.

10 Varkarakis MJ, Gaeta J, Moore RH, Murphy GP. Superficial bladder tumor. Aspects of clinical progression. Urology. 1974;4:414-20.

11 Edwards TJ, Dickinson AJ, Natale S, Gosling J, McGrath JS. A prospective analysis of the diagnostic yield resulting from the attendance of 4020 patients at a protocol-driven haematuria clinic. BJU Int. 2006;97:301-5.

12 Hiatt RA, Ordonez JD. Dipstick urinalysis screening, asymptomatic microhematuria, and subsequent urological cancers in a population-based sample. Cancer Epidemiol Biomarkers Prev. 1994;3:439-43.

13 Abt D, Bywater M, Engeler DS, Schmid HP. Therapeutic options for intractable hematuria in advanced bladder cancer. Intern J Urol. 2013;20:651-60.

14 Fahmy NM, Mahmud S, Aprikian AG. Delay in the surgical treatment of bladder cancer and survival: systematic review of the literature. Eur Urol. 2006;50:1176-82.

15 Edwards TJ, Dickinson AJ, Gosling J, McInerney PD, Natale S, McGrath JS. Patient-specific risk of undetected malignant disease after investigation for haematuria, based on a 4-year follow-up. BJU Int. 2011;107:247-52.

16 Suh J, Jung JH, Jeong CW, Kwak C, Kim HH, $\mathrm{Ku} \mathrm{JH}$. Clinical significance of pre-treated neutrophil-lymphocyte ratio in the management of urothelial carcinoma: a systemic review and meta-analysis. Front Oncol. 2019;9: 1365 .
17 Antoniewicz A, Zapala L, Poletajew S, Borówka A. Macroscopic haematuria-a leading urological problem in patients on anticoagulant therapy: is the common diagnostic standard still advisable? ISRN Urol. 2012;2012:710734.

18 Wallis CJD, Juvet T, Lee Y, Matta R, Herschorn S, Kodama R, et al. Association between use of antithrombotic medication and hematuria-related complications. J Am Med Assoc. 2017;318:1260-71.

19 Gore JL, Lai J, Setodji CM, Litwin MS, Saigal CS. Mortality increases when radical cystectomy is delayed more than 12 weeks: results from a surveillance, epidemiology, and end results-medicare analysis. Cancer. 2009;115: 988-96.

20 Sullivan JG, Mcgrath J, Macdermott JP. Air insufflation as an adjunct to flexible cystoscopy. BJU Int. 2002 Dec;90:e46-8. Available from:

21 Ciudin A, Diaconu MG, Gosalbez D, Peri L, Garcia-Cruz E, Franco A, et al. Air cystoscopy is superior to water cystoscopy for the diagnosis of active hematuria. J Urol. 2013;190: 2097-101.

22 Bolenz C, Schröppel B, Eisenhardt A, SchmitzDräger BJ, Grimm MO. The investigation of hematuria. Dtsch Arztebl Int. 2018;115:801807.

23 Suarez-Ibarrola R, Hein S, Farin E, Waldbillig F, Kriegmair MC, Ritter M, et al. Current Standards in the Endoscopic Management of Bladder Cancer: A Survey Evaluation among Urologists in German-Speaking Countries. Urol Int. 2020;104(5-6):410-6.
Findings of Flexible Cystoscopy in the Setting of Acute Hematuria
Urol Int 2022;106:147-153

DOI: $10.1159 / 000517374$ 